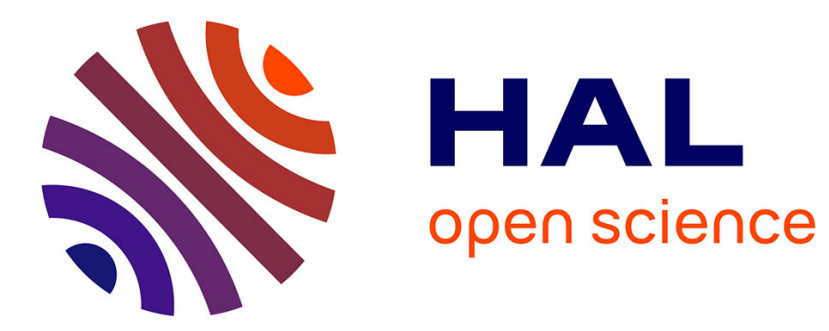

\title{
Variance reduction in stochastic homogenization : proof of concept, using antithetic variables
}

Ronan Costaouec, Claude Le Bris, Frédéric Legoll

\section{To cite this version:}

Ronan Costaouec, Claude Le Bris, Frédéric Legoll. Variance reduction in stochastic homogenization: proof of concept, using antithetic variables. [Research Report] RR-7207, Inria. 2010, pp.1-21. inria00457946

\section{HAL Id: inria-00457946 \\ https://hal.inria.fr/inria-00457946}

Submitted on 19 Feb 2010

HAL is a multi-disciplinary open access archive for the deposit and dissemination of scientific research documents, whether they are published or not. The documents may come from teaching and research institutions in France or abroad, or from public or private research centers.
L'archive ouverte pluridisciplinaire HAL, est destinée au dépôt et à la diffusion de documents scientifiques de niveau recherche, publiés ou non, émanant des établissements d'enseignement et de recherche français ou étrangers, des laboratoires publics ou privés. 
INSTITUT NATIONAL DE RECHERCHE EN INFORMATIQUE ET EN AUTOMATIQUE

\title{
Variance reduction in stochastic homogenization: proof of concept, using antithetic variables
}

\author{
Ronan Costaouec — Claude Le Bris — Frédéric Legoll
}

\section{$\mathbf{N}^{\circ} \mathbf{7 2 0 7}$}

February 2010

Thème NUM 



\title{
RIN RIA
}

\section{Variance reduction in stochastic homogenization: proof of concept, using antithetic variables}

\author{
Ronan Costaouec $^{* \dagger}$, Claude Le Bris ${ }^{* \dagger}$, Frédéric Legoll ${ }^{\ddagger \dagger}$ \\ Thème NUM — Systèmes numériques \\ Projet MICMAC
}

Rapport de recherche $n^{\circ} 7207$ - February 2010 - 21 pages

\begin{abstract}
We show that we can reduce the variance in a simple problem of stochastic homogenization using the classical technique of antithetic variables. The setting, and the presentation, are deliberately kept elementary. We point out the main issues, show some illustrative results, and demonstrate, both theoretically and numerically, the efficiency of the approach on simple cases.
\end{abstract}

Key-words: stochastic homogenization, variance reduction, antithetic variables

* CERMiCS, Ecole Nationale des Ponts et Chaussées, 6 et 8 avenue Blaise Pascal, Cité Descartes, 77455 Marne-la-Vallée Cedex 2, France. Contact: \{costaour,lebris\}@cermics.enpc.fr

$\dagger$ INRIA Rocquencourt, MICMAC team-project, Domaine de Voluceau, B.P. 105, 78153 Le Chesnay Cedex, France.

$\ddagger$ Institut Navier, LAMI, Ecole Nationale des Ponts et Chaussées, 6 et 8 avenue Blaise Pascal, Cité Descartes, 77455 Marne-la-Vallée Cedex 2, France. Contact: legoll@lami.enpc.fr 


\section{Réduction de variance en homogénéisation stochastique: l'exemple des variables antithétiques}

Résumé : Dans ce travail, nous mettons en oeuvre une technique de réduction de variance dans le cadre de l'homogénéisation stochastique. Plus précisément, nous montrons qu'il est possible de réduire la variance de la matrice homogénéisée calculée numériquement, en utilisant la technique des variables antithétiques. Nous avons volontairement choisi de nous placer dans un cadre de travail simple, afin d'identifier les principales difficultés. Nous démontrons, à la fois théoriquement et numériquement, l'efficacité de l'approche, dans des cas simples.

Mots-clés : homogénéisation stochastique, réduction de variance, variables antithétiques 


\section{Introduction}

Several settings in homogenization require the solution of corrector problems posed on the entire space $\mathbb{R}^{d}$. In practice, truncations of these problems over bounded domains are considered and the homogenized coefficients are obtained in the limit of large domains. The question arises to accelerate such computations. In the deterministic case, acceleration techniques reminiscent from signal filtering have been introduced in [5]. The work has since then been significantly improved in [12]. In [5], it was shown that acceleration techniques efficient for deterministic problems do not necessarily perform well in the stochastic framework. In the latter case, the main difficulty is related to the intrinsic noise present in the simulation. The challenge is consequently not that much to improve the rate of convergence, which is intrinsically that of the central limit theorem, but rather to reduce the variance, thereby improving the prefactor of the convergence given by the central limit theorem. Although very well investigated in other application fields such as financial mathematics, variance reduction techniques seem to have not been applied to the context of stochastic homogenization. The purpose of the present contribution is to present a first attempt in reducing the variance in stochastic homogenization. For this purpose, we consider a simple situation, and a simple variance reduction technique. The probability theoretic arguments we will make use of are elementary. The equation under consideration is a simple elliptic equation in divergence form, with a scalar coefficient. The coefficient is assumed to consist of independent, identically distributed random variables set on a simple mesh (see (2) below). The technique used for variance reduction is that of antithetic variables. Our setting is academic in nature, somewhat far from physically relevant cases, and elementary. Many more difficult situations could be addressed: other types of stationary ergodic coefficients, matrix rather than scalar coefficients, other types of equations, other techniques for variance reduction, ... The present contribution is a proof of concept: variance reduction can be achieved in stochastic homogenization. Future works $[3,4,11]$ will provide more details on the numerics and the theory, and also address some of the many possible extensions mentioned above.

\section{Stochastic homogenization theory}

Although we wish to keep the mathematical formalism as limited as possible in our exposition, we need to introduce the basic setting of stochastic homogenization (see [16] for a similar presentation and related issues). Throughout this article, $(\Omega, \mathcal{F}, \mathbb{P})$ is a probability space and we denote by $\mathbb{E}(X)=\int_{\Omega} X(\omega) d \mathbb{P}(\omega)$ the expectation value of any random variable $X \in L^{1}(\Omega, d \mathbb{P})$. We next fix $d \in \mathbb{N}^{*}$ (the ambient physical dimension), and assume that the group $\left(\mathbb{Z}^{d},+\right)$ acts on $\Omega$. We denote by $\left(\tau_{k}\right)_{k \in \mathbb{Z}^{d}}$ this action, and assume that it preserves the measure $\mathbb{P}$, that is, for all $k \in \mathbb{Z}^{d}$ and all $A \in \mathcal{F}, \mathbb{P}\left(\tau_{k} A\right)=\mathbb{P}(A)$. We assume that the action $\tau$ is ergodic, that is, if $A \in \mathcal{F}$ is such that $\tau_{k} A=A$ for any $k \in \mathbb{Z}^{d}$, then $\mathbb{P}(A)=0$ or 1 . In addition, we define the following notion of stationarity (see [7]): any $F \in L_{\text {loc }}^{1}\left(\mathbb{R}^{d}, L^{1}(\Omega)\right)$ is said to be stationary if, for all $k \in \mathbb{Z}^{d}$,

$$
F(x+k, \omega)=F\left(x, \tau_{k} \omega\right),
$$


almost everywhere in $x$ and almost surely. In this setting, the ergodic theorem [15, 17] can be stated as follows: Let $F \in L^{\infty}\left(\mathbb{R}^{d}, L^{1}(\Omega)\right)$ be a stationary random variable in the above sense. For $k=\left(k_{1}, k_{2}, \ldots k_{d}\right) \in \mathbb{Z}^{d}$, we set $|k|_{\infty}=\sup _{1 \leq i \leq d}\left|k_{i}\right|$. Then

$$
\frac{1}{(2 N+1)^{d}} \sum_{|k|_{\infty} \leq N} F\left(x, \tau_{k} \omega\right) \underset{N \rightarrow \infty}{\longrightarrow} \mathbb{E}(F(x, \cdot)) \quad \text { in } L^{\infty}\left(\mathbb{R}^{d}\right), \text { almost surely. }
$$

This implies that (denoting by $Q$ the unit cube in $\mathbb{R}^{d}$ )

$$
F\left(\frac{x}{\varepsilon}, \omega\right) \underset{\varepsilon \rightarrow 0}{*} \mathbb{E}\left(\int_{Q} F(x, \cdot) d x\right) \quad \text { in } L^{\infty}\left(\mathbb{R}^{d}\right) \text {, almost surely. }
$$

Besides technicalities, the purpose of the above setting is simply to formalize that, even though realizations may vary, the function $F$ at point $x \in \mathbb{R}^{d}$ and the function $F$ at point $x+k, k \in \mathbb{Z}^{d}$, share the same law. In the homogenization context we now turn to, this means that the local, microscopic environment (encoded in the coefficient $a$, see (3) below) is everywhere the same on average. From this, homogenized, macroscopic properties will follow.

We now fix an open, regular, bounded subset $\mathcal{D}$ of $\mathbb{R}^{d}$, an $L^{2}$ function $f$ on $\mathcal{D}$, and a random function $a$ assumed stationary in the sense (1) defined above. We also assume $a$ is bounded, positive and almost surely bounded away from zero. For simplicity, we take $a$ a random piecewise constant function of the form:

$$
a(x, \omega)=\sum_{k \in \mathbb{Z}^{d}} \mathbf{1}_{Q+k}(x) a_{k}(\omega)
$$

where $Q$ is the unit cube of $\mathbb{R}^{d}$ and $\left(a_{k}(\omega)\right)_{k \in \mathbb{Z}^{d}}$ denotes a family of i.i.d. random variables. The standard results of stochastic homogenization [2, 14] apply to the boundary value problem

$$
\left\{\begin{aligned}
-\operatorname{div}\left(a\left(\frac{x}{\varepsilon}, \omega\right) \nabla u^{\varepsilon}\right) & =f \text { in } \mathcal{D} \\
u^{\varepsilon} & =0 \text { on } \partial \mathcal{D} .
\end{aligned}\right.
$$

These results state that, in the limit $\varepsilon \longrightarrow 0$, the homogenized problem obtained from $(3)$ reads:

$$
\left\{\begin{aligned}
-\operatorname{div}\left(A^{\star} \nabla u^{\star}\right) & =f \text { in } \mathcal{D} \\
u^{\star} & =0 \text { on } \partial \mathcal{D} .
\end{aligned}\right.
$$

The homogenized matrix $A^{\star}$ is defined as

$$
\left[A^{\star}\right]_{i j}=\mathbb{E}\left(\int_{Q}\left(e_{i}+\nabla w_{e_{i}}(y, \cdot)\right)^{T} a(y, \cdot)\left(e_{j}+\nabla w_{e_{j}}(y, \cdot)\right) d y\right),
$$


where, for any $p \in \mathbb{R}^{d}, w_{p}$ is the solution (unique up to the addition of a (random) constant) in $\left\{w \in L_{\text {loc }}^{2}\left(\mathbb{R}^{d}, L^{2}(\Omega)\right), \quad \nabla w \in L_{\text {unif }}^{2}\left(\mathbb{R}^{d}, L^{2}(\Omega)\right)\right\}$ to

$$
\left\{\begin{array}{l}
-\operatorname{div}\left[a(y, \omega)\left(p+\nabla w_{p}(y, \omega)\right)\right]=0 \text { a.s. on } \mathbb{R}^{d}, \\
\nabla w_{p} \text { is stationary in the sense of }(1), \\
\mathbb{E}\left(\int_{Q} \nabla w_{p}(y, \cdot) d y\right)=0
\end{array}\right.
$$

where we have used the notation $L_{\text {unif }}^{2}$ for the uniform $L^{2}$ space, that is the space of functions for which, say, the $L^{2}$ norm on a ball of unit size is bounded above independently from the center of the ball.

The solution $u^{\varepsilon}$ to (3) is known to converge to the solution $u^{\star}$ to (4) in various appropriate senses. The tensor and function $A^{\star}$ and $u^{\star}$ are deterministic quantities, although they originate from a series of random problems. This is a consequence of the ergodic setting described above, which allows random microscopic quantities to average out in deterministic macroscopic quantities. Note however that the computation of $A^{\star}$ requires the computation of the so-called corrector functions $w_{p}$, which are random.

The above result generalizes that of the classical periodic setting (see e.g. [2, 9]) where, instead of being stationary ergodic, the function $a$ in (3) is periodic. Then, although the homogenized problem can be expressed similarly, the crucial difference is that (at least in this simple linear case) the corrector problem can, in the periodic case, be reduced to the equation $-\operatorname{div}\left[a(y)\left(p+\nabla w_{p}(y)\right)\right]=0$ set on the periodic cell $Q=[0,1]^{d}$, and not on the entire space $\mathbb{R}^{d}$ as in (6). Correspondingly, the terms of the homogenized tensor in (5) are simple deterministic integrals on $Q$. In the random case, equation (6) is intrinsically set on the entire space and the numerical approximation of the solution $w_{p}$ to the corrector problem (6) is the main computational challenge. Problem (6) is in practice truncated on a bounded domain $Q_{N}=[-N, N]^{d}$ and usually supplied with periodic boundary conditions:

$$
\left\{\begin{array}{l}
-\operatorname{div}\left(a(\cdot, \omega)\left(p+\nabla w_{p}^{N}(\cdot, \omega)\right)\right)=0 \quad \text { on } \quad Q_{N}, \\
w_{p}^{N} \text { is } Q_{N} \text {-periodic. }
\end{array}\right.
$$

Correspondingly, we set:

$$
\left[A_{N}^{\star}\right]_{i j}(\omega)=\frac{1}{\left|Q_{N}\right|} \int_{Q_{N}}\left(e_{i}+\nabla w_{e_{i}}^{N}(y, \omega)\right)^{T} a(y, \omega)\left(e_{j}+\nabla w_{e_{j}}^{N}(y, \omega)\right) d y .
$$

In the limit of large domains $Q_{N}$, the homogenized tensor (5) is recovered. In addition, the rate of convergence with which the truncated values approach the exact homogenized value $A^{\star}$ can be assessed theoretically. We refer to $[8,18]$ for the proof of all the above statements. As will be seen below, the variance of the random variables involved plays a role in the approximation procedure. Reducing this variance is the problem we now consider. 


\section{Variance reduction}

\subsection{Classical Monte Carlo method}

As mentioned above, the large size (large $N$ ) limit of the coefficient (8) obtained using the solution of the truncated corrector problem (7) gives the value of the homogenized coefficient (5). Formally, this is a convergence of the type $A_{N}^{\star}(\omega) \longrightarrow A^{\star}$ as $N \longrightarrow+\infty$ almost surely in $\Omega$. The practical approach to this problem is the Monte-Carlo approach. We now briefly investigate the role of the variance in the problem.

To start with, we briefly consider the one-dimensional setting. Although this setting is very particular (and sometimes misleading because oversimplified), it also allows to already understand the basic features of the problem and the bottom line of the approach, with the economy of many unnecessary technicalities.

In the one-dimensional setting, the definition (2) reads

$$
a(x, \omega)=\sum_{k \in \mathbb{Z}} \mathbf{1}_{[k, k+1[}(x) a_{k}(\omega)
$$

with $\left(a_{k}(\omega)\right)_{k \in \mathbb{Z}}$ a family of i.i.d. random variables. It is easily seen that the truncated corrector problem (7) can be explicitly solved and leads to the value

$$
a_{N}^{\star}(\omega)=\left(\frac{1}{2 N} \sum_{k=-N}^{N-1} \frac{1}{a_{k}(\omega)}\right)^{-1}
$$

of the approximation for the homogenized tensor (here, a scalar coefficient of course). In the limit of large $N$, it almost surely converges to the value of the exact homogenized coefficient

$$
a^{\star}=\mathbb{E}\left(\frac{1}{a_{0}}\right)^{-1}
$$

This exact value is readily obtained explicitly solving (5)-(6). The simplest possible argument consists now in considering $\left(a_{N}^{\star}(\omega)\right)^{-1}=\frac{1}{2 N} \sum_{k=-N}^{N-1} \frac{1}{a_{k}(\omega)}$ and remark that the rate of convergence of this quantity to $\left(a^{\star}\right)^{-1}$ is evidently given by the central limit theorem, where the variance of the random variable $\left(a_{k}(\omega)\right)^{-1}$ plays a crucial role. Although correct, this argument exploits too much the very peculiar nature of the one-dimensional setting (we have taken the inverse of the coefficient and recasted it as a sum, a fact that is not possible otherwise than in one dimension). An argument with slightly more generality consists in considering $a_{N}^{\star}(\omega)$ itself - and not its inverse-, and, using elementary calculus, showing that it also converges to $a^{\star}$ with a rate of convergence where the variance of $a_{0}(\omega)$ again plays the crucial role. Indeed, one may for instance remark that 
$\mathbb{E}\left(\left|\left(\frac{1}{2 N} \sum_{k=-N}^{N-1} \frac{1}{a_{k}}\right)^{-1}-\mathbb{E}\left(\frac{1}{a_{0}}\right)^{-1}\right|^{2}\right)$ may be bounded from above (using a simple almost sure upper bound of $\left.a_{k}(\omega)\right)$ by $\mathbb{E}\left(\left|\left(\frac{1}{2 N} \sum_{k=-N}^{N-1} \frac{1}{a_{k}}\right)-\mathbb{E}\left(\frac{1}{a_{0}}\right)\right|^{2}\right)$ up to an irrelevant multiplicative constant and that the latter quantity, once easily computed, is of the form $\frac{1}{2 N} \operatorname{Var}\left(\frac{1}{a_{0}}\right)$. Again, the variance of the random coefficient plays a role.

In dimensions higher than one, the situation is considerably more intricate and the rate of convergence with which the coefficient arising from the truncated computation converges to its limit is not so simple to evaluate. This is the purpose, under appropriate conditions (called mixing conditions and which are indeed met in our present setting), of the work [8].

The numerical practice is as follows. A set of $M$ independent realizations of the random coefficient $a$ are considered. The corresponding truncated problems (7) are solved, and an empirical mean of the truncated coefficients (8) is inferred. This empirical mean only agrees with the theoretical value of the truncated coefficient within a margin of error which is given by the central limit theorem (in terms of $M$ ). The variance of the coefficients therefore again plays a role, as a prefactor. For a sufficiently large truncation size $N$, this truncated value is admitted to be the exact value of the coefficients. The error made is controled by the estimations of the theoretical work [8]. Of course, the overall computation described above is expensive, because each realization requires a new solution to the $d$-dimensional boundary value problem (7) of presumably large a size since $N$ is taken large. There is therefore a huge interest in reducing the cost of the computation, or, otherwise stated, in reaching a better accuracy at a given computational cost. Since the variance of the truncated homogenized tensor is an important ingredient, reducing the variance becomes a challenging and sensitive issue.

More explicitly, let $\left(a^{\mathbf{m}}(x, \omega)\right)_{1<\mathbf{m}<M}$ denote $M$ independent and identically distributed underlying random fields. We define a family $\left(A_{N}^{\star, \mathbf{m}}\right)_{1 \leq \mathbf{m} \leq M}$ of i.i.d. homogenized matrices by, for any $1 \leq i, j \leq d$,

$$
\left[A_{N}^{\star, \mathbf{m}}\right]_{i j}(\omega)=\frac{1}{\left|Q_{N}\right|} \int_{Q_{N}}\left(e_{i}+\nabla w_{e_{i}}^{N, \mathbf{m}}(\cdot, \omega)\right)^{T} a^{\mathbf{m}}(\cdot, \omega)\left(e_{j}+\nabla w_{e_{j}}^{N, \mathbf{m}}(\cdot, \omega)\right),
$$

where $w_{e_{j}}^{N, \mathbf{m}}$ is the solution of the corrector problem associated to $a^{\mathbf{m}}$. Then we define for each component of $A_{N}^{\star}$ the empirical mean and variance

$$
\begin{aligned}
\mu_{M}\left(\left[A_{N}^{\star}\right]_{i j}\right) & =\frac{1}{M} \sum_{\mathbf{m}=1}^{M}\left[A_{N}^{\star, \mathbf{m}}\right]_{i j}, \\
\sigma_{M}\left(\left[A_{N}^{\star}\right]_{i j}\right) & =\frac{1}{M-1} \sum_{\mathbf{m}=1}^{M}\left(\left[A_{N}^{\star, \mathbf{m}}\right]_{i j}-\mu_{M}\left(\left[A_{N}^{\star}\right]_{i j}\right)\right)^{2} .
\end{aligned}
$$

Since the matrices $A_{N}^{\star, \mathbf{m}}$ are i.i.d., the strong law of large numbers applies:

$$
\mu_{M}\left(\left[A_{N}^{\star}\right]_{i j}\right)(\omega) \underset{M \rightarrow+\infty}{\longrightarrow} \mathbb{E}\left(\left[A_{N}^{\star}\right]_{i j}\right) \text { almost surely. }
$$


The central limit theorem then yields

$$
\sqrt{M}\left(\mu_{M}\left(\left[A_{N}^{\star}\right]_{i j}\right)-\mathbb{E}\left(\left[A_{N}^{\star}\right]_{i j}\right)\right) \underset{M \rightarrow+\infty}{\stackrel{\mathcal{L}}{\longrightarrow}} \sqrt{\operatorname{Var}\left(\left[A_{N}^{\star}\right]_{i j}\right)} \mathcal{N}(0,1),
$$

where the convergence holds in law, and $\mathcal{N}(0,1)$ denotes the standard gaussian law. Introducing its 95 percent quantile, it is standard to consider that the exact mean $\mathbb{E}\left(\left[A_{N}^{\star}\right]_{i j}\right)$ is equal to $\mu_{M}\left(\left[A_{N}^{\star}\right]_{i j}\right)$ within a margin of error $1.96 \frac{\sqrt{\operatorname{Var}\left(\left[A_{N}^{\star}\right]_{i j}\right)}}{\sqrt{M}}$. The exact variance $\operatorname{Var}\left(\left[A_{N}^{\star}\right]_{i j}\right)$ being unknown in practice, it is customary to replace it by the empirical variance given in (12) above. It is therefore considered that the expectation $\mathbb{E}\left(\left[A_{N}^{\star}\right]_{i j}\right)$ lies in the interval

$$
\left[\mu_{M}\left(\left[A_{N}^{\star}\right]_{i j}\right)-1.96 \frac{\sqrt{\sigma_{M}\left(\left[A_{N}^{\star}\right]_{i j}\right)}}{\sqrt{M}}, \mu_{M}\left(\left[A_{N}^{\star}\right]_{i j}\right)+1.96 \frac{\sqrt{\sigma_{M}\left(\left[A_{N}^{\star}\right]_{i j}\right)}}{\sqrt{M}}\right]
$$

The value $\mu_{M}\left(\left[A_{N}^{\star}\right]_{i j}\right)$ is thus, for both $M$ and $N$ sufficiently large, adopted as the approximation of the exact value $\left[A^{\star}\right]_{i j}$.

Of course, a tensorial argument could be applied here, not considering separately each entry of the matrix but treating the matrix as a whole. The approach developed above, component by component, is sufficient for the simple cases considered in the present work.

\subsection{Antithetic variable for stochastic homogenization}

We know from the previous section that constructing empirical means approximating $\mathbb{E}\left(A_{N}^{\star}\right)$ with a smaller variance at the same computational cost is of high interest. We now describe a possible approach to achieve this goal.

In generality, fix $M=2 \mathcal{M}$. Suppose that we have $\mathcal{M}$ i.i.d. copies $\left(a^{\mathbf{m}}(x, \omega)\right)_{1 \leq \mathbf{m} \leq \mathcal{M}}$ of $a(x, \omega)$. Construct next $\mathcal{M}$ i.i.d. antithetic random fields

$$
b^{\mathbf{m}}(x, \omega)=T\left(a^{\mathbf{m}}(x, \omega)\right), \quad 1 \leq \mathbf{m} \leq \mathcal{M},
$$

from the $\left(a^{\mathbf{m}}(x, \omega)\right)_{1<\mathbf{m}<\mathcal{M}}$. The map $T$ transforms the random field $a^{\mathbf{m}}$ into another, socalled antithetic, field $b^{\mathbf{m}}$. Explicit examples of such $T$ are given in the sequel (see (20) and Section 4 below). The transformation is performed in such a way that, for each $\mathbf{m}, b^{\mathbf{m}}$ should have the same law as $a^{\mathbf{m}}$, namely the law of the coefficient $a$. Somewhat vaguely stated, if the coefficient $a$ was obtained in a coin tossing game (using a fair coin), then $b^{\mathbf{m}}$ would be head each time $a^{\mathbf{m}}$ is tail and vice versa. We refer the reader to Figure 1 below for 
explicit illustrative examples of such a construction. Then, for each $1 \leq \mathbf{m} \leq \mathcal{M}$, we solve two corrector problems. One is associated to the original $a^{\mathbf{m}}$, the other one is associated to the antithetic field $b^{\mathbf{m}}$. Using its solution $v_{p}^{N, \mathbf{m}}$, we define the antithetic homogenized matrix $B_{N}^{\star, \mathbf{m}}$, whose elements read, for $1 \leq i, j \leq d$,

$$
\left[B_{N}^{\star, \mathbf{m}}\right]_{i j}(\omega)=\frac{1}{\left|Q_{N}\right|} \int_{Q_{N}}\left(e_{i}+\nabla v_{e_{i}}^{N, \mathbf{m}}(\cdot, \omega)\right)^{T} b^{\mathbf{m}}(\cdot, \omega)\left(e_{j}+\nabla v_{e_{j}}^{N, \mathbf{m}}(\cdot, \omega)\right) .
$$

And finally we set, for any $1 \leq \mathbf{m} \leq \mathcal{M}$,

$$
\widetilde{A}_{N}^{\star, \mathbf{m}}(\omega):=\frac{1}{2}\left(A_{N}^{\star, \mathbf{m}}(\omega)+B_{N}^{\star, \mathbf{m}}(\omega)\right) .
$$

Since $a^{\mathbf{m}}$ and $b^{\mathbf{m}}$ are identically distributed, so are $A_{N}^{\star, \mathbf{m}}$ and $B_{N}^{\star, \mathbf{m}}$. Thus, $\widetilde{A}_{N}^{\star, \mathbf{m}}$ is unbiased (that is, $\left.\mathbb{E}\left(\widetilde{A}_{N}^{\star, \mathbf{m}}\right)=\mathbb{E}\left(A_{N}^{\star, \mathbf{m}}\right)\right)$. In addition, it satisfies:

$$
\widetilde{A}_{N}^{\star, \mathbf{m}} \underset{N \rightarrow+\infty}{\longrightarrow} A^{\star} \text { almost surely, }
$$

because $b$ is ergodic.

Let us define new estimators

$$
\begin{aligned}
\mu_{\mathcal{M}}\left(\left[\widetilde{A}_{N}^{\star}\right]_{i j}\right) & =\frac{1}{\mathcal{M}} \sum_{\mathbf{m}=1}^{\mathcal{M}}\left[\widetilde{A}_{N}^{\star, \mathbf{m}}\right]_{i j}, \\
\sigma_{\mathcal{M}}\left(\left[\widetilde{A}_{N}^{\star}\right]_{i j}\right) & =\frac{1}{\mathcal{M}-1} \sum_{\mathbf{m}=1}^{\mathcal{M}}\left(\left[\widetilde{A}_{N}^{\star, \mathbf{m}}\right]_{i j}-\mu_{\mathcal{M}}\left(\left[\widetilde{A}_{N}^{\star}\right]_{i j}\right)\right)^{2},
\end{aligned}
$$

which require $2 \mathcal{M}$ resolutions of corrector problems, i.e. as many as the classical estimators (12), since we choose $M=2 \mathcal{M}$. In addition, note that we have built a new random variable whose variance is

$$
\operatorname{Var}\left(\left[\widetilde{A}_{N}^{\star}\right]_{i j}\right)=\frac{1}{2} \operatorname{Var}\left(\left[A_{N}^{\star}\right]_{i j}\right)+\frac{1}{2} \mathbb{C o v}\left(\left[A_{N}^{\star}\right]_{i j},\left[B_{N}^{\star}\right]_{i j}\right) .
$$

Applying the central limit theorem to $\widetilde{A}_{N}^{\star}$, we obtain

$$
\sqrt{\mathcal{M}}\left(\mu_{\mathcal{M}}\left(\left[\widetilde{A}_{N}^{\star}\right]_{i j}\right)-\mathbb{E}\left(\left[A_{N}^{\star}\right]_{i j}\right)\right) \underset{\mathcal{M} \rightarrow+\infty}{\stackrel{\mathcal{L}}{\longrightarrow}} \sqrt{\operatorname{Var}\left(\left[\widetilde{A}_{N}^{\star}\right]_{i j}\right)} \mathcal{N}(0,1) .
$$

Similarly to (14), we deduce a confidence interval from this convergence. The exact mean $\mathbb{E}\left(\left[\widetilde{A}_{N}^{\star}\right]_{i j}\right)$ is equal to $\mu_{\mathcal{M}}\left(\left[\widetilde{A}_{N}^{\star}\right]_{i j}\right)$ within a margin of error $1.96 \frac{\sqrt{\operatorname{Var}\left(\left[\widetilde{A}_{N}^{\star}\right]_{i j}\right)}}{\sqrt{\mathcal{M}}}$. It results from (17) that, if

$$
\operatorname{Cov}\left(\left[A_{N}^{\star}\right]_{i j},\left[B_{N}^{\star}\right]_{i j}\right) \leq 0,
$$


then the width of this interval has been diminished by the new approach, and, correspondingly, the quality of approximation at given computational cost has increased.

To understand slightly more in details at the theoretical level why the approach is likely to perform well, we again consider the one-dimensional setting (9) for which we recall the explicit expressions (10) and (11) for the truncated and the exact homogenized coefficients, respectively.

Suppose as a first illustration that $a_{0}$ is a Bernoulli distributed random variable $a_{0} \sim$ $\mathcal{B}(1 / 2)$ :

$$
\mathbb{P}\left(a_{0}=\alpha\right)=1 / 2 \text { and } \mathbb{P}\left(a_{0}=\beta\right)=1 / 2,
$$

for some $0<\alpha<\beta$. Defining the antithetic variable

$$
b_{k}(\omega)=\alpha+\beta-a_{k}(\omega)
$$

and next the antithetic field

$$
b(x, \omega)=\sum_{k \in \mathbb{Z}} \mathbf{1}_{[k, k+1[}(x) b_{k}(\omega)=\sum_{k \in \mathbb{Z}} \mathbf{1}_{[k, k+1[}(x)\left(\alpha+\beta-a_{k}(\omega)\right),
$$

it is immediately seen that

$$
\frac{1}{2}\left(\frac{1}{a_{N}^{\star}(\omega)}+\frac{1}{b_{N}^{\star}(\omega)}\right)=\mathbb{E}\left(\frac{1}{a_{0}}\right) .
$$

The variance of the inverse of the truncated coefficient has vanished. This example might seem oversimplified because we are indeed making use of two peculiarities of the problem: the set $\{\alpha, \beta\}$ of values taken by the coefficient $a$ has cardinality two, and the explicit expression (10) allows us to explicitly manipulate the inverse of the homogenized coefficient. The situation, although oversimplified, is yet a first good indicator of the interest of the approach. As in the previous section, we can be slightly more general, by considering for instance that the random coefficient $a$ is now uniformly distributed over a given interval, say $a_{0} \sim \mathcal{U}([\alpha, \beta])$. Then,

$$
\frac{1}{2}\left(\frac{1}{a_{N}^{\star}(\omega)}+\frac{1}{b_{N}^{\star}(\omega)}\right)=\frac{1}{2 N} \sum_{k=-N}^{N-1} \frac{1}{2}\left(\frac{1}{a_{k}(\omega)}+\frac{1}{b_{k}(\omega)}\right) .
$$

It is a simple matter to show that, because the function $x \mapsto 1 / x$ is decreasing, we have

$$
\operatorname{Cov}\left(\frac{1}{a_{0}}, \frac{1}{b_{0}}\right) \leq 0 \text {. }
$$

Consider indeed a decreasing function $f$, and $X$ and $Y$ two independent random variables, identically distributed according to $\mathcal{U}([\alpha, \beta])$. Since $x \mapsto f(\alpha+\beta-x)$ is increasing, we observe that

$$
(f(X)-f(Y))(f(\alpha+\beta-X)-f(\alpha+\beta-Y)) \leq 0,
$$


hence

$$
\mathbb{E}[f(X) f(\alpha+\beta-X)] \leq \mathbb{E}[f(X)] \mathbb{E}[f(\alpha+\beta-X)],
$$

which reads $\operatorname{Cov}[f(X), f(\alpha+\beta-X)] \leq 0$. Choosing $f(x)=1 / x$ yields $(22)$.

Since

$$
\operatorname{Var}\left(\frac{1}{2}\left(\frac{1}{a_{N}^{\star}}+\frac{1}{b_{N}^{\star}}\right)\right)=\frac{1}{4 N} \operatorname{Var}\left(\frac{1}{a_{0}}\right)+\frac{1}{4 N} \operatorname{Cov}\left(\frac{1}{a_{0}}, \frac{1}{b_{0}}\right),
$$

we conclude that

$$
\operatorname{Var}\left(\frac{1}{2}\left(\frac{1}{a_{N}^{\star}}+\frac{1}{b_{N}^{\star}}\right)\right) \leq \operatorname{Var}\left(\frac{1}{a_{2 N}^{\star}}\right) .
$$

Therefore, $\mathbb{E}\left(1 / a_{0}\right)$ can be approximated either by $(21)$ or by $1 / a_{2 N}^{\star}$, with an equal cost (i.e. an equal number of random variables in both sums), but the former has less variance than the latter. It is hence of better quality.

As mentioned above, the practice in dimensions higher than one is to generate a set of identically distributed coefficients for each truncated corrector problem, and to use (15). The appropriate analogous one-dimensional approach is to consider $\mathcal{M}=\frac{M}{2}$ independent copies of $a(x, \omega)$ and set

$$
\begin{aligned}
\widetilde{a}_{N}^{\star, \mathbf{m}}(\omega) & :=\frac{1}{2}\left(a_{N}^{\star, \mathbf{m}}(\omega)+b_{N}^{\star, \mathbf{m}}(\omega)\right) \\
& =\frac{1}{2}\left(\frac{1}{2 N} \sum_{k=-N}^{N-1} \frac{1}{a_{k}^{\mathbf{m}}(\omega)}\right)^{-1}+\frac{1}{2}\left(\frac{1}{2 N} \sum_{k=-N}^{N-1} \frac{1}{b_{k}^{\mathbf{m}}(\omega)}\right)^{-1}
\end{aligned}
$$

with empirical mean

$$
\mu_{\mathcal{M}}\left(\widetilde{a}_{N}^{\star}\right)(\omega)=\frac{1}{\mathcal{M}} \sum_{\mathbf{m}=1}^{\mathcal{M}} \widetilde{a}_{N}^{\star, \mathbf{m}}(\omega) .
$$

We approach more generality since

$$
\mu_{\mathcal{M}}\left(\widetilde{a}_{N}^{\star}\right)(\omega) \underset{\mathcal{M} \rightarrow+\infty}{\longrightarrow} \mathbb{E}\left(\widetilde{a}_{N}^{\star}\right)=\mathbb{E}\left(a_{N}^{\star}\right) \text { almost surely }
$$

but $\mathbb{E}\left(a_{N}^{\star}\right) \neq a^{\star}$. It can again be remarked that $a_{N}^{\star}(\omega)$ is an increasing function of the uniform variables $\left(a_{k}(\omega)\right)_{k \in \mathbb{Z}}$. From this observation, it is possible to show that $\operatorname{Cov}\left(a_{N}^{\star}, b_{N}^{\star}\right) \leq$ 0 , and to conclude that the variance of $\mu_{\mathcal{M}}\left(\widetilde{a}_{N}^{\star}\right)$ is smaller than that of $\mu_{2 \mathcal{M}}\left(a_{N}^{\star}\right)$. For this proof on a model by analogy, as well as for proofs that variance reduction is indeed achieved for some actual settings in dimensions higher than one (such as for instance those from $[1,10,6])$, we refer to $[3,11]$. The above simplified arguments were only meant to have pedagogic value. 


\section{Numerical experiments}

The previous section provides some elementary ingredients for a theoretical analysis of the efficiency of the approach. The one-dimensional setting is however too particular. More convincing theoretical arguments have to be developed. As announced, this will be the purpose of future publications. Meanwhile, it is possible to test the approach on actual two-dimensional cases, and this is the purpose of this section to report on such tests. As above, we only consider random coefficients that are piecewise constant and of the form (2). The test cases we choose to consider correspond to three different laws for $a_{0}$ :

- case (i): a Bernoulli law of parameter $1 / 2$, namely $a_{0} \sim \mathcal{B}(1 / 2), \mathbb{P}\left(a_{0}=\alpha\right)=1 / 2$ and $\mathbb{P}\left(a_{0}=\beta\right)=1 / 2$;

- case (ii): a Bernoulli law of parameter $1 / 3$, namely $a_{0} \sim \mathcal{B}(1 / 3), \mathbb{P}\left(a_{0}=\alpha\right)=1 / 3$ and $\mathbb{P}\left(a_{0}=\beta\right)=2 / 3$

- case (iii): a uniform law, namely $a_{0} \sim \mathcal{U}([\alpha, \beta])$.

We take the specific values $\alpha=3$ and $\beta=20$, just to fix the ideas. Similar qualitative conclusions would be reached with other generic values. Figure 1 shows a realization of $a$ and its antithetic field $b$ in cases (i) and (iii).

Our numerical tests have been performed using the finite elements software FreeFem ++ developed by F. Hecht (Paris VI, see [13]). The discretization of the corrector problem is performed using $\mathbb{P} 1$ Lagrange finite elements, and a regular $Q$-periodic mesh of $Q_{N}$. The discretization meshsize is fixed and has value $h=0.2$.

It is worth mentioning how we practically proceed to generate an antithetic variable. This may indeed be delicate. We have taken random coefficients that can all originally be expressed in terms of a uniformly distributed random variable (with a view, notably, to be consistent with the way a random variable is practically generated on a computer). We then build the antithetic variable precisely using the 'mother' uniform random variable. The technique is best explained on case (ii). Write the variable $a_{0} \sim \mathcal{B}(1 / 3)$ as $a_{0} \sim$ $\alpha+(\beta-\alpha) \mathbf{1}_{\left\{1 / 3 \leq U_{0} \leq 1\right\}}$ where $U_{0} \sim \mathcal{U}([0,1])$ denotes a random variable that has uniform law on the interval $[0,1]$. The antithetic variable is then taken as $b_{0} \sim \alpha+(\beta-\alpha) \mathbf{1}_{\left\{0 \leq U_{0} \leq 2 / 3\right\}}$ and the correspondence is made realization by realization using the actual realization of $U_{0}$.

In cases (i) and (ii), in dimension 2, the exact homogenized tensor is known to be isotropic, $A^{\star}=a^{\star} \mathbb{I}_{2}$ (see [14, Chap. 7, pp. 234-237] for a proof). Of course, for $N$ finite, $A_{N}^{\star}$ is a generic matrix, but our numerical experiments consistently show that, for $N$ sufficiently large, the off-diagonal terms are very small on average compared to the diagonal terms, in the three cases we have considered. Table 1 summarizes, in case (iii), the estimated means and variances of the components of $A_{N}^{\star}$ for different values of $N$. It confirms that the main sources of variance are the diagonal terms. The same conclusion holds in cases (i) and (ii).

In our three test cases, we have compared for different values of $N$ the estimated variance of $\left[\widetilde{A}_{N}^{\star}\right]_{11}$ with that of $\left[A_{N}^{\star}\right]_{11}$. In order to quantitatively assess the efficiency of the antithetic 

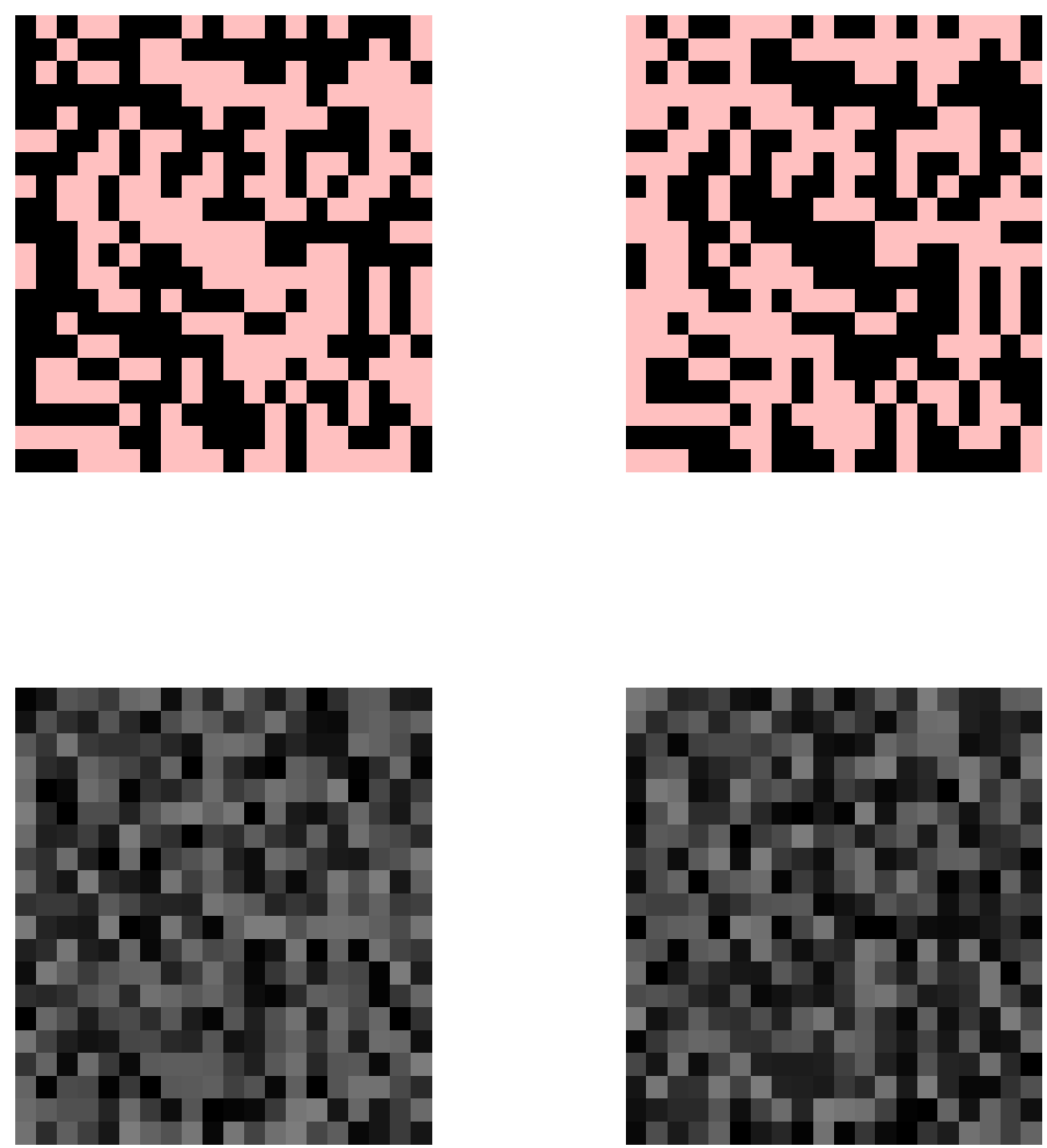

Figure 1: Realization of $a(x, \omega)$ given by (2) (left) and the associated antithetic field $b(x, \omega)$ (right). Top figures: $a_{0} \sim \mathcal{B}(1 / 2)$; bottom figures: $a_{0} \sim \mathcal{U}([\alpha, \beta])$. 


\begin{tabular}{l|ccc}
$N$ & {$\left[A_{N}^{\star}\right]_{11}$} & {$\left[A_{N}^{\star}\right]_{22}$} & {$\left[A_{N}^{\star}\right]_{12}$} \\
\hline 5 & $10.42(0.370)$ & $10.39(0.385)$ & $0.00391(0.00555)$ \\
10 & $10.39(0.0724)$ & $10.39(0.0747)$ & $0.00369(0.00110)$ \\
20 & $10.37(0.0292)$ & $10.37(0.0262)$ & $0.00089(0.00031)$ \\
40 & $10.39(0.00471)$ & $10.39(0.00487)$ & $-0.00219(0.00009)$ \\
60 & $10.38(0.00201)$ & $10.38(0.00203)$ & $0.00059(0.00005)$ \\
80 & $10.38(0.00101)$ & $10.38(0.00119)$ & $0.00013(0.00002)$ \\
100 & $10.38(0.00077)$ & $10.38(0.00076)$ & $0.00010(0.00001)$ \\
\hline
\end{tabular}

Table 1: For each entry of $A_{N}^{\star}$, empirical mean $\mu_{100}\left(\left[A_{N}^{\star}\right]_{i j}\right)$ (and empirical variance $\sigma_{100}\left(\left[A_{N}^{\star}\right]_{i j}\right)$, in brackets), in the case (iii).

variables method, we introduce the effectivity ratio

$$
R\left(\left[A_{N}^{\star}\right]_{11}\right)=\frac{\sigma_{100}\left(\left[A_{N}^{\star}\right]_{11}\right)}{2 \sigma_{50}\left(\left[\widetilde{A}_{N}^{\star}\right]_{11}\right)} .
$$

The factor 2 at the denominator accounts for the number of realizations associated to the classical and antithetic Monte-Carlo methods, given that we wish to work at fixed computational cost. Indeed, after solving $M=2 \mathcal{M}$ corrector problems (7), one can either build a confidence interval of size $1.96 \sqrt{\sigma_{M}\left(\left[A_{N}^{\star}\right]_{11}\right) / M}$ following (13) and (14), or a confidence interval of size $1.96 \sqrt{\sigma_{\mathcal{M}}\left(\left[\widetilde{A}_{N}^{\star}\right]_{11}\right) / \mathcal{M}}$ following (18).

Our next table, Table 2, contains the values of this representative ratio for each test case. We have also plotted on Figure 2 the curves of estimated means (12) and (16), with their confidence intervals, for the three cases under study here.

If we admit that the theory developed in the previous section applies to the twodimensional case, another manner to check variance reduction is to compute the empirical covariance between $\left[A_{N}^{\star}\right]_{11}$ and $\left[B_{N}^{\star}\right]_{11}$ (recall (19)). This is the reason why we have also plotted on Figure 2 the normalized empirical value of this covariance,

$$
\frac{\operatorname{Cov}\left(\left[A_{N}^{\star}\right]_{11},\left[B_{N}^{\star}\right]_{11}\right)}{\sqrt{\operatorname{Var}\left(\left[A_{N}^{\star}\right]_{11}\right) \operatorname{Var}\left(\left[B_{N}^{\star}\right]_{11}\right)}}
$$

for test case (iii) (similar results have been obtained for the two other test cases).

The results are self-explanatory: the variance is reduced. The reduction is not spectacular, but it is definite, and, equally importantly, systematic. Considering that the approach induces no additional computational cost at all, this is very good. Other more adapted, but also more delicate to design and implement, variance reduction approaches will be tested in the future $[4,11]$, and one may expect even more significant reductions. 


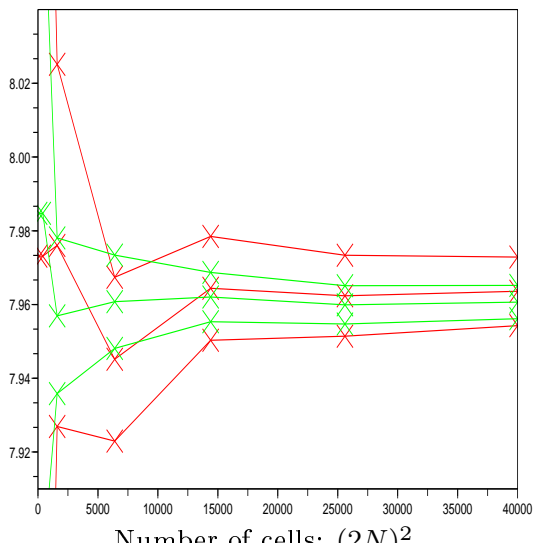

Number of cells: $(2 N)^{2}$

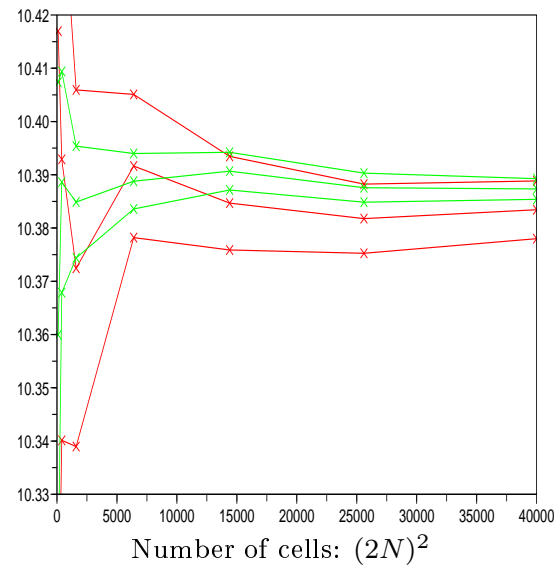

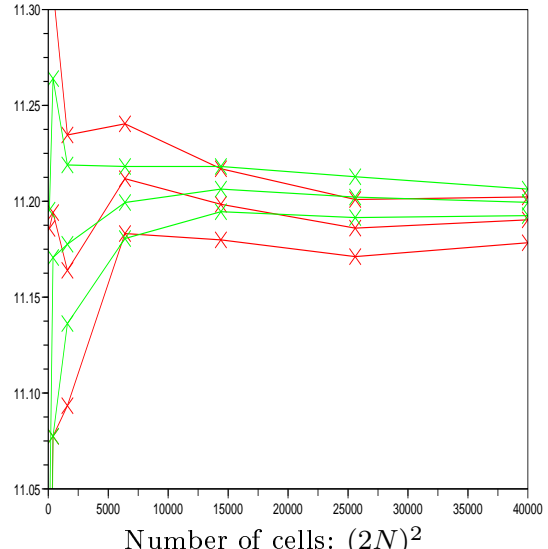

Number of cells: $(2 N)^{2}$

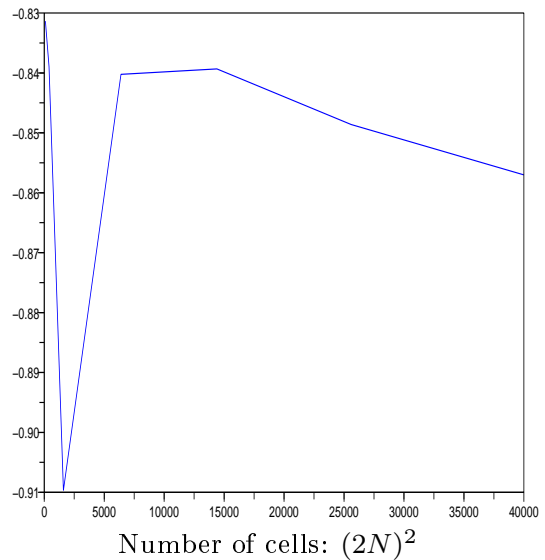

Figure 2: Estimated means (with confidence intervals) for $\left[A_{N}^{\star}\right]_{11}$ (red) and $\left[\widetilde{A}_{N}^{\star}\right]_{11}$ (green), in the cases $a_{0} \sim \mathcal{B}(1 / 2)$ (top left), $a_{0} \sim \mathcal{B}(1 / 3)$ (top right) and $a_{0} \sim \mathcal{U}([\alpha, \beta]$ ) (bottom left). In the latter case, we also plot the estimator (23) of the normalized covariance between $\left[A_{N}^{\star}\right]_{11}$ and $\left[B_{N}^{\star}\right]_{11}$ (bottom right). 


\begin{tabular}{l|ccc}
$N$ & $a_{0} \sim \mathcal{B}(1 / 2)$ & $a_{0} \sim \mathcal{B}(1 / 3)$ & $a_{0} \sim \mathcal{U}([\alpha, \beta])$ \\
\hline 5 & 5.34 & 2.06 & 6.31 \\
10 & 3.91 & 1.56 & 6.46 \\
20 & 5.41 & 2.92 & 10.2 \\
40 & 3.07 & 2.31 & 6.67 \\
60 & 4.41 & 2.47 & 6.16 \\
80 & 4.49 & 1.95 & 5.68 \\
100 & 4.28 & 2.99 & 7.89 \\
\hline
\end{tabular}

Table 2: Representative effectivity ratios $R\left(\left[A_{N}^{\star}\right]_{11}\right)$ for test cases (i), (ii) and (iii). The number shown gives the gain in computational time or, equivalently, at given computational cost, the square of the gain in the width of the confidence interval.

\section{Variance reduction for the solution $u^{\star}$}

We conclude this article examining the problem of variance reduction from a slightly different perspective. We have so far investigated the question of variance reduction for the homogenized tensor $A^{\star}$. This is the question typically relevant in Mechanics, where for instance determining the homogenized tensor is an important issue because it allows to define, say, the Young modulus or the Poisson ratio of the homogenized material. In some contexts however, the focus is more on the solution of the homogenized problem, rather than on the coefficients of the homogenized equation. For a given right-hand side $f$ in (3) (or for a set of such right-hand sides), one wishes to know the behaviour of the solution $u^{\varepsilon}$ for small $\varepsilon$. Now, reducing the variance on the solution $u$ is not exactly the same question as reducing the variance on the coefficients of the equation (because the map that associates the solution to the coefficients of the equation is a highly nonlinear nonlocal map). Note also that a systematic way to investigate the question would of course be to study the variance of the homogenized operator itself (or of its eigenelements) and this is indeed on our agenda to do so in a more extensive article $[4,11]$. But for the time being, we briefly mention here a possible variance reduction approach on the solution $u^{\star}$, for a given representative right-hand side $f$.

In principle, one may think of several possible ways for computing the solution $u^{\star}$ to the homogenized problem (4). A first approach, which we denote by (M1), consists in the following schematic sequence of computations

$$
\left(a^{\mathbf{m}}(x, \omega)\right)_{1 \leq \mathbf{m} \leq M} \stackrel{\text { corrector }}{\longrightarrow}\left(A_{N}^{\star, \mathbf{m}}(\omega)\right)_{1 \leq \mathbf{m} \leq M} \stackrel{\frac{1}{M} \sum}{\longrightarrow} \mu_{M}\left(A_{N}^{\star}\right) \stackrel{(24)}{\longrightarrow} u_{N, M}^{\star},
$$

where $u_{N, M}^{\star}$ solves the boundary value problem

$$
\left\{\begin{aligned}
-\operatorname{div}\left(\mu_{M}\left(A_{N}^{\star}\right)(\omega) \nabla u_{N, M}^{\star}(x, \omega)\right) & =f \text { in } \mathcal{D} \\
u_{N, M}^{\star}(x, \omega) & =0 \text { on } \partial \mathcal{D} .
\end{aligned}\right.
$$


In short, (M1) consists in first approximating $A^{\star}$ using the Monte Carlo approach and its outcome $\mu_{M}\left(A_{N}^{\star}\right)$, and next to solve for $u_{N, M}^{\star}$.

A second approach, (M2), consists in the sequence

$$
\left(a^{\mathbf{m}}(x, \omega)\right)_{1 \leq \mathbf{m} \leq M} \stackrel{\text { corrector }}{\longrightarrow} \mathrm{pb}\left(A_{N}^{\star, \mathbf{m}}(\omega)\right)_{1 \leq \mathbf{m} \leq M} \stackrel{(25)}{\longrightarrow}\left(u_{N}^{\star, \mathbf{m}}(\cdot, \omega)\right)_{1 \leq \mathbf{m} \leq M} .
$$

Otherwise stated, for each $1 \leq \mathbf{m} \leq M$, the problem

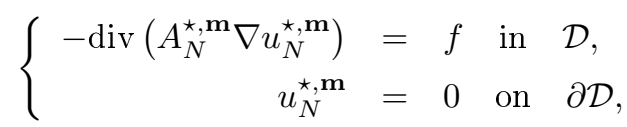

is first solved, and the empirical mean and variance of the corresponding solutions are constructed:

$$
\begin{aligned}
\mu_{M}\left(u_{N}^{\star}\right)(x, \omega) & =\frac{1}{M} \sum_{\mathbf{m}=1}^{M} u_{N}^{\star, \mathbf{m}}(x, \omega), \\
\sigma_{M}\left(u_{N}^{\star}\right)(x, \omega) & =\frac{1}{M-1} \sum_{\mathbf{m}=1}^{M}\left(u_{N}^{\star, \mathbf{m}}(x, \omega)-\mu_{M}\left(u_{N}^{\star}\right)(x, \omega)\right)^{2} .
\end{aligned}
$$

The empirical mean is then taken as the approximation of our seeked solution $u^{\star}$.

Of course, it is immediately seen that a set of approaches, intermediate between (M1) and (M2), can be designed. This is the set of approaches (M3). For each $1 \leq \mathbf{m} \leq M$, we first solve the corrector problem, and thus obtain $A_{N}^{\star, \mathbf{m}}(\omega)$. We next set $M=P R$, and define, for each $1 \leq \mathbf{r} \leq R$,

$$
\mu_{P}^{\mathbf{r}}\left(A_{N}^{\star}\right)(\omega)=\frac{1}{P} \sum_{\mathbf{p}=1}^{P} A_{N}^{\star, \mathbf{p}+(\mathbf{r}-1) P}(\omega),
$$

which is an empirical mean computed with $P$ realizations among the $M$ available realizations. For each $1 \leq \mathbf{r} \leq R$, we next solve the boundary value problem

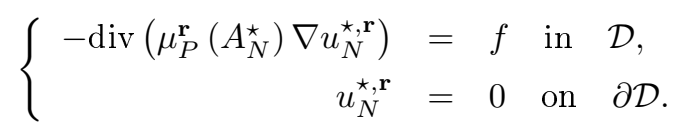

The estimators for $u^{\star}$ then are

$$
\begin{aligned}
& \mu_{R, P}\left(u_{N}^{\star}\right)(x, \omega)=\frac{1}{R} \sum_{\mathbf{r}=1}^{R} u_{N}^{\star, \mathbf{r}}(x, \omega), \\
& \sigma_{R, P}\left(u_{N}^{\star}\right)(x, \omega)=\frac{1}{R-1} \sum_{\mathbf{r}=1}^{R}\left(u_{N}^{\star, \mathbf{r}}(x, \omega)-\mu_{R, P}\left(u_{N}^{\star}\right)(x, \omega)\right)^{2} .
\end{aligned}
$$


We observe that, in dimension one, the solution of (25) satisfies

$$
\left(u_{N}^{\star, \mathbf{m}}\right)^{\prime}(x, \omega)=-\frac{1}{a_{N}^{\star, \mathbf{m}}(\omega)}\left(F(x)-\frac{1}{|\mathcal{D}|} \int_{\mathcal{D}} F\right),
$$

where $F(x)$ is such that $F^{\prime}(x)=f(x)$. Hence, in view of (10) and (11), we have

$$
\mathbb{E}\left[\left(u_{N}^{\star, \mathbf{m}}\right)^{\prime}\right]=-\frac{1}{a^{\star}}\left(F(x)-\frac{1}{|\mathcal{D}|} \int_{\mathcal{D}} F\right)=\mathbb{E}\left[\left(u^{\star}\right)^{\prime}\right] .
$$

As a consequence, the empirical mean built following approach (M2), namely $\mu_{M}\left(u_{N}^{\star}\right)(x, \omega)$ defined by (26), is an unbiased estimator of $u^{\star}(x)$, for any finite $N$ and $M$, in the onedimensional case. The estimators built following approaches (M1) and (M3) do not share this property.

In the present work, we only consider approach (M2), leaving the study of the other approaches for future works. We apply the exact same technique as above, considering antithetic variables to reduce the variance. The variance under consideration is however now that of the approximation of $u^{\star}$.

We consider the test case (iii) defined in the previous section. We choose the right-hand side $f(x, y)=(x-0.5)^{2}+(y-0.5)^{2}$ on the domain $\mathcal{D}=Q=[0,1]^{2}$ (similar results have been obtained with other right-hand sides). The efficiency of the antithetic variable technique is assessed using the following ratio

$$
R\left(u_{N}^{\star}\right)=\inf _{x \in \mathcal{D}} \frac{\sigma_{100}\left(u_{N}^{\star}\right)}{2 \sigma_{50}\left(\widetilde{u}_{N}^{\star}\right)} .
$$

We have also checked that the technique does not introduce any bias by monitoring the estimator

$$
\sup _{x \in \mathcal{D}}\left|\frac{\mu_{100}\left(u_{N}^{\star}\right)-\mu_{50}\left(\widetilde{u}_{N}^{\star}\right)}{\mu_{100}\left(u_{N}^{\star}\right)}\right| .
$$

Numerical results are gathered in Table 3 . We observe that the technique does not introduce any bias, and that, again, a significant variance reduction, at fixed computational cost, is obtained. 


\begin{tabular}{l|cc}
$N$ & Estimator $(28)$ & Estimator $(27)$ \\
\hline 5 & $4.20 \times 10^{-4}$ & 10.1 \\
10 & $3.80 \times 10^{-4}$ & 10.9 \\
20 & $1.56 \times 10^{-3}$ & 14.6 \\
40 & $4.05 \times 10^{-4}$ & 11.8 \\
80 & $5.21 \times 10^{-4}$ & 9.10 \\
100 & $3.24 \times 10^{-4}$ & 9.02 \\
\hline
\end{tabular}

Table 3: Estimator (28) of the bias, and estimator (27) of the variance reduction, in the case $a_{0} \sim \mathcal{U}([\alpha, \beta])$ (the equation (25) has been solved on a mesh of size $h=0.1$ ).

Acknowledgments: The content of this article has been presented by the second author as a plenary lecture at the XXI CEDYA - XI CMA international conference, September 21-25, 2009, Ciudad Real. The authors thank Xavier Blanc for several stimulating discussions. Part of this work was initiated while the second author was visiting the Institute for Mathematics and its Applications and the Department of Mathematics of the University of Minnesota. The hospitality of these institutions is gratefully acknowledged. The work of the authors is partially supported by ONR under contract Grant 00014-09-1-0470.

\section{References}

[1] A. Anantharaman and C. Le Bris, Homogenization of a weakly randomly perturbed periodic material, C. R. Acad. Sci. Série I, 2009, submitted.

[2] A. Bensoussan, J.-L. Lions, and G. Papanicolaou, Asymptotic analysis for periodic structures, Studies in Mathematics and its Applications, 5. North-Holland Publishing Co., Amsterdam-New York, 1978.

[3] X. Blanc, R. Costaouec, C. Le Bris, and F. Legoll, Variance reduction in stochastic homogenization using antithetic variables, Markov Processes and Related Fields, in preparation.

[4] X. Blanc, R. Costaouec, C. Le Bris, and F. Legoll, Lect. Notes Comput. Sci. Eng., Springer, in preparation.

[5] X. Blanc and C. Le Bris, Improving on computation of homogenized coefficients in the periodic and quasi-periodic settings, Netw. Heterog. Media, 2010, in press.

[6] X. Blanc, C. Le Bris, and P.-L. Lions, Une variante de la théorie de l'homogénéisation stochastique des opérateurs elliptiques [A variant of stochastic homogenization theory for elliptic operators], C. R. Acad. Sci. Série I, 343:717-724, 2006. 
[7] X. Blanc, C. Le Bris, and P.-L. Lions, Stochastic homogenization and random lattices, J. Math. Pures Appl., 88:34-63, 2007.

[8] A. Bourgeat and A. Piatnitski, Approximation of effective coefficients in stochastic homogenization, Ann I. H. Poincaré - PR, 40:153-165, 2004.

[9] D. Cioranescu and P. Donato, An introduction to homogenization, Oxford Lecture Series in Mathematics and its Applications, 17. Oxford University Press, New York, 1999.

[10] R. Costaouec, C. Le Bris, and F. Legoll, Approximation numérique d'une classe de problèmes en homogénéisation stochastique [Numerical approximation of a class of problems in stochastic homogenization], C. R. Acad. Sci. Série I, 348(1-2):99-103, 2010.

[11] R. Costaouec, Université Paris Est, Ph.D thesis, in preparation.

[12] A. Gloria and F. Otto, An optimal error estimate in stochastic homogenization of discrete elliptic equations, in preparation.

[13] FreeFEM, http://freefem++.org

[14] V. V. Jikov, S. M. Kozlov, and O. A. Oleinik, Homogenization of differential operators and integral functionals, Springer-Verlag, 1994.

[15] U. Krengel, Ergodic theorems, de Gruyter Studies in Mathematics, vol. 6, de Gruyter, 1985 .

[16] C. Le Bris, Some numerical approaches for "weakly" random homogenization, Proceedings of ENUMATH 2009, Lect. Notes Comput. Sci. Eng., Springer, in press.

[17] A. N. Shiryaev, Probability, Graduate Texts in Mathematics, vol. 95, Springer, 1984.

[18] V. V. Yurinskii, Averaging of symmetric diffusion in random medium, Sibirskii Mat. Zh., 27(4):167-180, 1986. 


\section{Contents}

1 Introduction $\quad 3$

2 Stochastic homogenization theory $\quad 3$

3 Variance reduction $\quad 6$

3.1 Classical Monte Carlo method . . . . . . . . . . . . . . . . . . 6

3.2 Antithetic variable for stochastic homogenization . . . . . . . . . . 8

4 Numerical experiments $\quad 12$

5 Variance reduction for the solution $u^{\star} \quad 16$ 
Unité de recherche INRIA Rocquencourt Domaine de Voluceau - Rocquencourt - BP 105 - 78153 Le Chesnay Cedex (France)

Unité de recherche INRIA Futurs : Parc Club Orsay Université - ZAC des Vignes 4, rue Jacques Monod - 91893 ORSAY Cedex (France)

Unité de recherche INRIA Lorraine : LORIA, Technopôle de Nancy-Brabois - Campus scientifique 615, rue du Jardin Botanique - BP 101 - 54602 Villers-lès-Nancy Cedex (France)

Unité de recherche INRIA Rennes : IRISA, Campus universitaire de Beaulieu - 35042 Rennes Cedex (France)

Unité de recherche INRIA Rhône-Alpes : 655, avenue de l'Europe - 38334 Montbonnot Saint-Ismier (France)

Unité de recherche INRIA Sophia Antipolis : 2004, route des Lucioles - BP 93 - 06902 Sophia Antipolis Cedex (France) 